\title{
Manufacturing of Bent Silicon Crystals for Steering of Particle Beam at Ultra-High Energy Synchrotrons ${ }^{+}$
}

\author{
Enrico Bagli ${ }^{1}$, Andrea Balbo ${ }^{2}$, Laura Bandiera ${ }^{1}$, Stefano Baricordi ${ }^{3}$, Riccardo Camattari ${ }^{1}$, \\ Sara Carturan 4,5, Davide Casotti ${ }^{1}$, Giuseppe Cruciani ${ }^{6}$, Davide De Salvador 4,5 , \\ Andrea Mazzolari ${ }^{1}$, Marco Romagnoni ${ }^{1,6}$, Melissa Tamisari ${ }^{1,7}, *$, Thu Nhi Tran Thi ${ }^{8}$ and \\ Jaap van den Berg ${ }^{9}$ \\ 1 INFN, Section of Ferrara, Via Saragat 1, 44122 Ferrara, Italy \\ 2 Department of Engeneering, University of Ferrara, Via Saragat 1, 44122 Ferrara, Italy \\ 3 Baricordi Stefano, Via Otello Putinati, 67 A 44123 Ferrara, Italy \\ 4 Department of Physics and Astronomy Galileo Galilei, University of Padova, Via Marzolo 8, \\ 35131 Padova, Italy \\ 5 INFN, Legnaro National Laboratory, Viale dell'Università, 235020 Legnaro (Padova), Italy \\ 6 Department of Physics and Earth Science, University of Ferrara, Via Saragat 1/c, 44122 Ferrara, Italy \\ 7 Department of Biomedical and Surgical Specialized Sciences, University of Ferrara, Via Saragat 1/c, \\ 44122 Ferrara, Italy \\ 8 European Synchrotron Radiation Facility (ESRF), 38000 Grenoble, France \\ 9 Department of Engineering and Technology School of Computing and Engineering, \\ University of Huddersfield, Huddersfield HD1 3DH, UK \\ * Correspondence: tamisari@fe.infn.it \\ + Presented at the 37th International Symposium on Dynamical Properties of Solids (DyProSo 2019), Ferrara, \\ Italy, 8-12 September 2019.
}

Published: 5 September 2019

Since the '70, physics of interaction between bent crystals and heavy particles (protons or ions) is investigated at various worldwide particle accelerators, driven by the possibility to use bent crystals as optical elements for steering or focusing charged particle beams.

Recent improvements in crystal manufacturing techniques, joined with a deeper understanding of the physics behind crystals-particle beams interactions lead to a discovery of numerous interactional effects appearing as crystal planes or axis are aligned to a charged particle beam. The technology readiness level is mature enough to allow the use of crystals even in the Large Hadron Collider (LHC) of CERN, where crystals are being experimented as primary collimators of the protons or ions circulating beam, and are suggested as core elements of fixed-target experiments. The crystals, that operates without the need of a power source, does not require a cryogenic environment and are extremely compact and lightweight, can represent a reliable alternative to the use of magnets or other optical elements in ultra-high energy and intensity particle modern particle accelerators.

The purpose of this work is describe manufacturing and characterization solutions of crystals suitable for installation in the LHC as primary collimators of the proton or lead ions circulating beams. Successful development of such crystals is based on a merging of ultra-modern technologies used in microelectronics, X-ray science, ultra-precise optical and mechanical machining. Availability of this innovative generation of crystals makes to envisage innovative possibilities for particle beam steering at ultra-high energy and intensities particle accelerators.

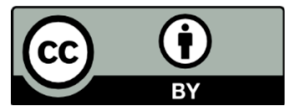

(C) 2019 by the authors. Licensee MDPI, Basel, Switzerland. This article is an open access article distributed under the terms and conditions of the Creative Commons Attribution (CC BY) license (http://creativecommons.org/licenses/by/4.0/). 